\title{
Failed export of the adrenocorticotrophin receptor from the endoplasmic reticulum in non-adrenal cells: evidence in support of a requirement for a specific adrenal accessory factor
}

\author{
L A Noon, J M Franklin, P J King, N J Goulding', L Hunyady² \\ and A J L Clark \\ Department of Endocrinology, Barts and the London, Queen Mary School of Medicine, West Smithfield, University of London, London EC1A 7BE, UK \\ ${ }^{1}$ Department of Biochemical Pharmacology, Barts and the London, Queen Mary School of Medicine, University of London, London, UK \\ ${ }^{2}$ Department of Physiology, Semmelweis University Faculty of Medicine, Budapest, Hungary \\ (Requests for offprints should be addressed to A J L Clark; Email: a.j.clark@qmul.ac.uk)
}

\begin{abstract}
Difficulty in expressing the adrenocorticotrophin (ACTH) receptor (melanocortin 2 receptor; $\mathrm{MC} 2 \mathrm{R}$ ) after transfection of various MC2R expression vectors has been experienced by many researchers. Reproducible evidence for expression has been obtained only in the Y6/OS3 corticoadrenal cell lines or in cells expressing endogenous melanocortin receptors. In order to determine the cause of this failure of expression we have undertaken the following studies. An MC2R expression plasmid was constructed in which the green fluorescent protein (GFP) coding region had been added to the $\mathrm{C}$-terminus of the mature protein. Transfection of this plasmid into Y6 cells with a cAMP-responsive reporter plasmid demonstrated normal function of this receptor. Imaging of $\mathrm{CHO}$ cells expressing
\end{abstract}

MC2R-GFP revealed perinuclear expression, although a cholecystokinin receptor (CCKR)-GFP construct was efficiently expressed at the cell surface. Y6 cells, in contrast, showed cell surface fluorescence after transfection with MC2R-GFP. Several other cell types showed a similar pattern of GFP distribution characteristic of retention in the endoplasmic reticulum. Counterstaining with an anti-KDEL antibody confirmed this location. Coexpression of the MC2R and the CCKR-GFP did not impair CCKR trafficking to the cell surface, implying a receptor-specific impairment to trafficking in the $\mathrm{CHO}$ cell which was absent in the Y6 cell.

Journal of Endocrinology (2002) 174, 17-25

\section{Introduction}

Adrenocorticotrophin (ACTH) is a 39 amino acid peptide, produced by the anterior pituitary gland, that exerts a steroidogenic effect on the cells of the adrenal cortex. These effects are mediated by a specific $G$ protein-coupled receptor, the melanocortin 2 receptor (MC2R) which signals predominantly through the cAMP pathway by stimulating adenylate cyclase activity. One of five melanocortin receptors, the MC2R, was cloned in 1992 and identified as the receptor for ACTH largely on the basis of its restricted localization to the adrenal cortex and its homology to other members of the melanocortin receptor family (Mountjoy et al. 1992). Its designation as the ACTH receptor was the subject of some initial controversy because of the difficulties encountered in trying to obtain convincing heterologous expression data. Limited expression studies were reported in Cloudman S91 cells but these results were complicated by the presence of an endogenous MC1 ( $\alpha$-melanocyte-stimulating hormone) receptor in this cell line (Mountjoy et al. 1992).
Subsequent examples of functional MC2R expression, again in Cloudman cells (Naville et al. 1996, Penhoat et al. 2000) and in COS-7 cells using a high efficiency transfection protocol (Weber et al. 1993), were likewise hindered by the presence of endogenous melanocortin receptors, as a consequence of which background function had to be subtracted from observed function in transfected cells. Many groups, including our own, have failed to obtain functional expression of the MC2R in cells that lack endogenous melanocortin receptors including HEK293, NIH3T3, CHO-K1 and NCI H295R cells and Xenopus oocytes (Naville et al. 1996 and authors' unpublished observations).

Satisfactory expression data has only been achievable in a single cell type to date, the Y6 or OS3 cell. These two cell lines were derived from MC2R-expressing Y1 adrenal cells, grown in the presence of forskolin, a treatment that has resulted in permanent failure to express the endogenous MC2R gene (Schimmer et al. 1995a). Although the nature of this impaired expression is incompletely understood (Schimmer et al. 1995b, Frigeri et al. 
2000), Y6 or OS3 cells transfected with MC2R expression constructs will express the receptor, allowing it to be studied without background interference. Two studies have now reported results with this system using cAMP generation studies and radioligand binding techniques (Yang et al. 1997, Elias et al. 1999) and this model has contributed to studies of MC2R desensitization (Baig et al. 2001). However, it is still not clear why MC2R expression is achievable only in a cell line as derived as the Y6/OS3 cell.

The aim of this study is to address this question of the nature of failed MC2R expression in non-adrenal cells. We have constructed a C-terminally tagged MC2R-GFP fusion protein and expressed it in a range of non-adrenal cell lines. This has enabled us to track the subcellular localization of the receptor for the first time. The use of GFP-fusion proteins has been extensively reviewed (Kallal \& Benovic 2000, Tavare et al. 2001) and is a method that has been employed successfully to study the cellular trafficking of many $G$ protein-coupled receptors (GPCRs), including the $\beta$-adrenergic (Barak et al. 1997), cholecystokinin (Tarasova et al. 1997) and vasopressin (Schulein et al. 1998) receptors, without interfering with the normal biological behaviour of the protein. Our findings suggest that there is a common causal explanation for failed functional expression in that receptor protein is specifically retarded in the endoplasmic reticulum of cell lines other than Y6. This implies the existence of a protein-specific transport system or chaperone in the adrenal cell type.

\section{Materials and Methods}

\section{Cell culture}

Reagents and media were obtained from Sigma (Poole, Dorset, UK) unless otherwise indicated. Y6 cells (a gift from Prof. B Schimmer) were grown in a 1:1 DMEM/ Ham's F10 media containing 15\% horse serum, 2.5\% fetal calf serum and $1 \%$ penicillin/streptomycin (Pen/Strep). CHO cells were cultured in Ham's F12 containing 10\% fetal calf serum and 1\% Pen/Strep.

\section{Expression constructs and transfection}

The C-terminally labelled MC2R-GFP fusion protein was made by cloning the mouse MC2R into pEGFP-N3 (Clontech, Cambridge, Cambs, UK). The cholecystokinin receptor (CCKR)-GFP fusion protein used as the positive control for cell surface expression was the kind gift of Dr S A Wank. The Golgi localization vector (pEYFP-Golgi) (Clontech) contains the N-terminal region of $\beta 1,4-$ galactosyltransferase which includes a peptide sequence that targets the fusion protein to the trans-medial region of the Golgi apparatus. Cytomegalovirus (CMV) renilla luciferase (Promega, Southampton, UK) was used to

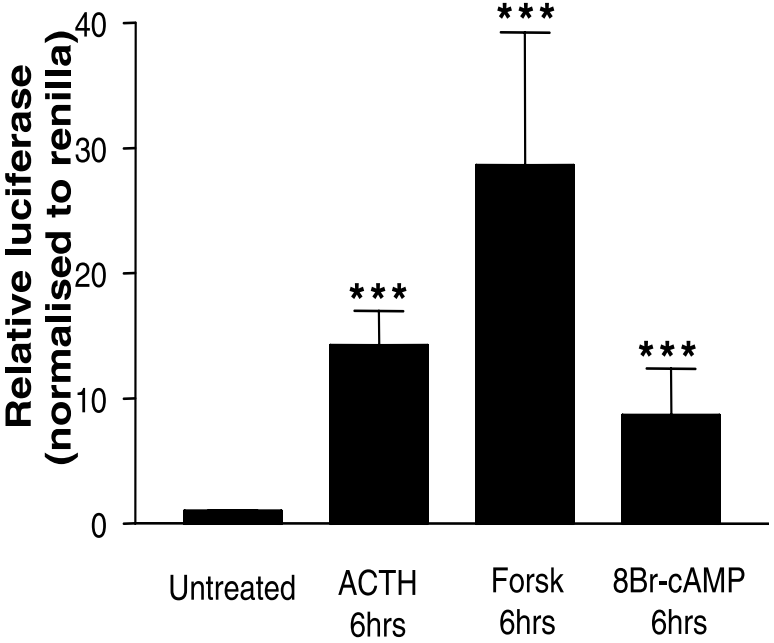

Figure 1 Response of $\mathrm{Y} 6$ cells stimulated with ACTH, forskolin (Forsk) and 8Br-cAMP for $6 \mathrm{~h}$. Cells were transfected $48 \mathrm{~h}$ prior to stimulation with the $\alpha \mathrm{GSU}-846$ luciferase reporter and the CMV renilla luciferase construct. Cells stimulated with $10^{-8} \mathrm{M} \mathrm{ACTH}$ were transfected with the MC2R-GFP while those stimulated with forskolin or $8 \mathrm{Br}$-cAMP were transfected with an equivalent amount of pcDNA3 (in order to balance the ratio of lipid to DNA in the transfection). Corrected luciferase was normalized with respect to the appropriate unstimulated control. $n=4$. *** $P<0 \cdot 001$.

correct luciferase activity for transfection efficiency. The cAMP luciferase reporter construct containing the -846 bp promoter region of the $\alpha$-glycoprotein subunit gene $(\alpha-G S U)$ was the kind gift of Prof. J Burrin (Burrin et al. 1998). Lipofection was used to transfect both $\mathrm{CHO}$ and Y6 cells grown to $80 \%$ confluence in 12-well plates using Lipofectamine Plus (Gibco Life Technologies, Paisley, Strathclyde, UK) in accordance with the manufacturer's guidelines.

\section{Luciferase assay}

Cells grown to $80 \%$ confluence were transfected with control (CMV renilla luciferase), reporter ( $\alpha$ GSU-846 luciferase) and test (MC2R-GFP, wild type mouse MC2R, or empty vector - pcDNA3) plasmid constructs $48 \mathrm{~h}$ prior to stimulation. The ratio of the three plasmids used was kept constant irrespective of the scale of the experiment or the transfection method used. In experiments using 12 -well plates $0 \cdot 25 \mu \mathrm{g}$ test $/ 0 \cdot 2 \mu \mathrm{g}$ reporter/ $0.05 \mu \mathrm{g}$ control DNA was used per well. These quantities were scaled up 20 -fold in $90-\mathrm{mm}$ plate experiments. Stimulations were performed in serum-free conditions for $6 \mathrm{~h}$ using $\mathrm{ACTH}\left[10^{-8} \mathrm{M}\right]$, forskolin $\left[10^{-5} \mathrm{M}\right]$ and 8Br-cAMP $\left[10^{-4} \mathrm{M}\right]$. Cell lysates were immediately harvested post-stimulation and assayed using the DualLuciferase Reporter Assay system (Promega). Luciferase 


\section{CCKR-GFP MC2R-GFP}

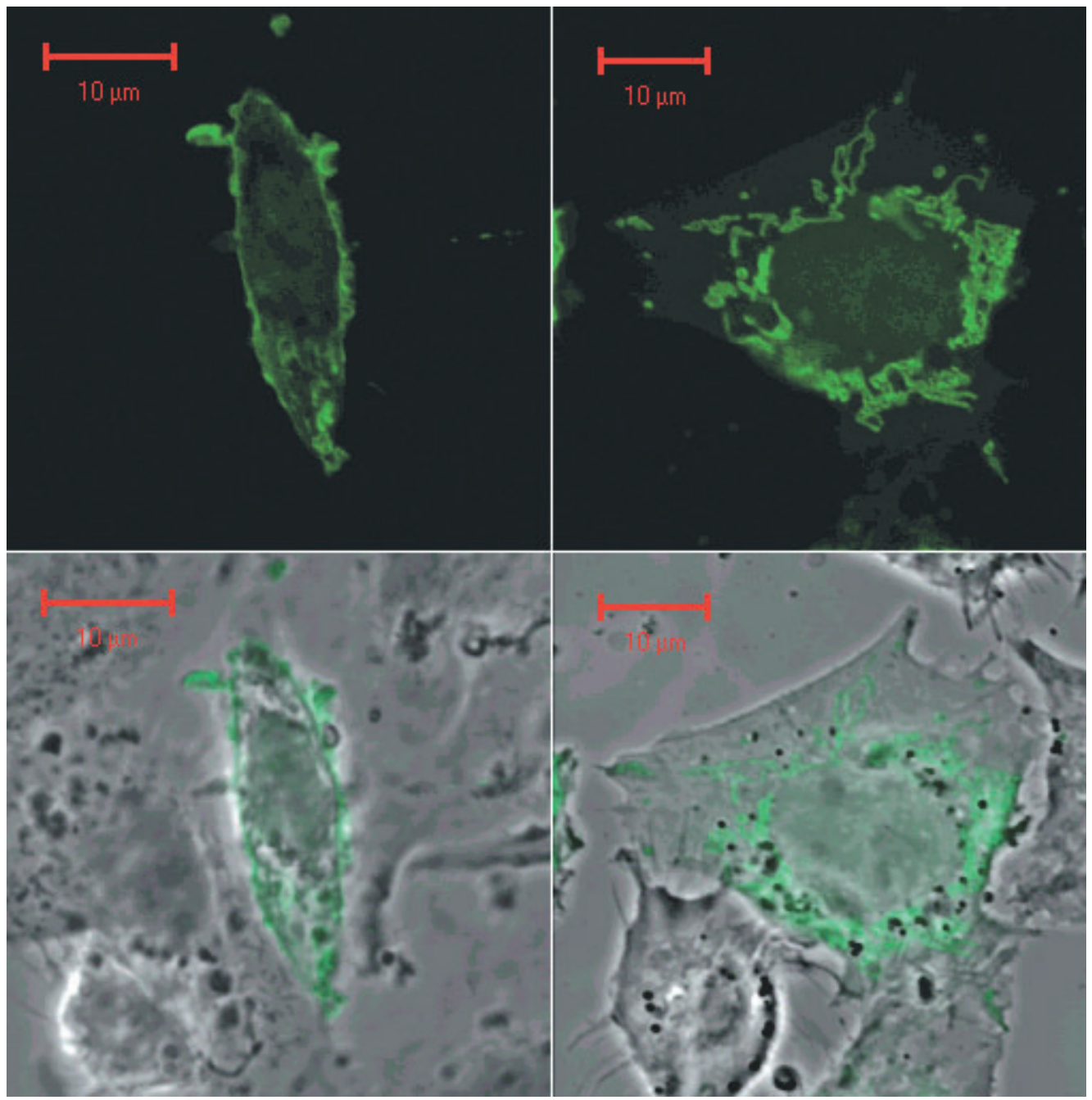

Figure 2 Confocal microscope images of $\mathrm{CHO}$ cells transiently expressing CCKR-GFP (left panels) or MC2R-GFP (right panels). Fluorescent images (top panels) were obtained by illumination at $488 \mathrm{~nm}$. These images are superimposed on top of a phase contrast image (lower panels).

activity was measured using a BioOrbit 1253 luminometer and values were normalized with respect to renilla luciferase activity.

\section{Microscopy}

Cells grown on glass coverslips were fixed and mounted $48 \mathrm{~h}$ post-transfection. Coverslips were washed twice in ice-cold PBS before being fixed in 4\% paraformaldehyde for $30 \mathrm{~min}$. The cells were then washed a further three times before being mounted in Fluoromount-G (Cambridge BioScience, Cambridge, Cambs, UK). Slides were stored at $4{ }^{\circ} \mathrm{C}$ until they were examined using either a Zeiss 510 or a Bio-Rad MRC600 or 1024 confocal microscope.

\section{Immunocytochemistry}

Endoplasmic reticulum localization was performed using a mouse monoclonal anti-KDEL antibody (SPA-827, Stressgen Biotechnologies Corporation, Victoria, Canada) as the primary antibody in cells permeabilized in Triton $\mathrm{X}-100$, and a Tetramethylrhodamine isothiocyanate (TRITC)-conjugated goat anti mouse secondary antibody. 


\section{Immunoblotting}

Whole cell extracts were prepared by the method described in Tolwinski et al. (1999) and Triton X-114 membrane extracts were prepared by the method described in Pryde (1998). SDS-PAGE was used to separate proteins on a $10 \%$ SDS ready gel (Biorad) and transfer to a nitrocellulose membrane was performed using semi-dry transfer apparatus. Membranes were blocked in $5 \%$ dried milk/ $0 \cdot 1 \%$ Tween PBS for $1 \mathrm{~h}$ before being probed with $1 / 1000$ anti-GFP (Roche) overnight at $4{ }^{\circ} \mathrm{C}$.

\section{Statistical analysis}

Statistical analysis of the luciferase results was performed using ANOVA.

\section{Results}

The cAMP luciferase assay provides an indirect but highly sensitive means for detecting the presence of a functioning receptor at the cell surface. The dependence of the $\alpha$ GSU-846 reporter activity on cAMP levels was demonstrated in the Y6 cell line by treatment with forskolin or the cAMP analogue 8Br-cAMP. These compounds, which mimic the effects of cAMP signalling, produced significant increases in luciferase activity of 28.6- and 8.7-fold respectively.

ACTH treatment of Y6 cells transiently expressing the MC2R modified by the addition of GFP (MC2R-GFP) resulted in a significant (14-fold) increase in luciferase activity compared with untreated cells expressing the same construct (Fig. 1). This result shows that the MC2R-GFP is capable of mediating a rise in cAMP in response to its ligand in the same manner as a functioning receptor. In a separate experiment, the performance of a wild type receptor and MC2R-GFP were compared under the same experimental conditions. Their responses indicate no impaired function of the fusion protein (data not shown). Our findings, therefore, demonstrate the preservation of normal function in the fusion protein despite the C-terminal tag, a fact that strongly implies that the protein is both correctly folded in, and targeted to, the cell membrane in Y6 cells.

Confocal laser scanning microscopy was used to explore the phenotypic characteristics of impaired expression in non-adrenal cells. A C-terminal GFP-tagged cholecystokinin receptor (CCKR), known to be trafficked to the cell surface in $\mathrm{CHO}$ cells, was used to demonstrate the ring-like localization of GFP around the perimeter of the cell that is characteristic of comprehensive cell surface expression (Fig. 2). This distribution was found to contrast sharply with that of GFP alone which when expressed had an intense but homogeneous nuclear and cytoplasmic localization (data not shown). The addition of GFP to the C-terminus of the MC2R resulted in a pattern of GFP localization in $\mathrm{CHO}$ cells that was distinct from that of the CCKR-GFP, in that fluorescence had a more discrete vesicular appearance with a predominantly perinuclear distribution (Fig. 2).

When SK-N-MC and HEK293 cells were transfected with the MC2R-GFP the pattern of fluorescence was found to be similar to that in $\mathrm{CHO}$ cells, suggesting that there may be a common cause for the failed functional expression of the MC2R (Fig. 3). In each case the fusion protein appeared to be successfully translated but rather than being membrane localized was sequestered in a perinuclear compartment of the cell. This pattern of fluorescence was very different from that found in Y6 cells transfected with the MC2R-GFP in which GFP appeared to be more closely associated with the membrane (Fig. 3).

The results of microscopy suggest that rather than following the normal route to the cell surface, the MC2RGFP accumulates behind a blockage in the secretory pathway of the cell. In order to investigate where in the secretory pathway the receptor was accumulating, immunostaining of permeabilized cells using an antiKDEL antibody was performed on $\mathrm{CHO}$ cells that had been transfected with the MC2R-GFP. This antibody identifies the resident endoplasmic reticulum proteins Grp78 (BiP) and Grp94 which possess the KDEL targeting sequence motif (Tang et al. 1993). Co-localization of the green and red fluorescent signals (shown as yellow in Fig. 4a) confirmed the build up of GFP in the endoplasmic reticulum of cells transfected with the MC2R-GFP. This localization does not exclude the Golgi however. Golgi localization was therefore undertaken using transient transfection of the EYFP-Golgi localization vector, and cells were imaged as before by confocal microscopy. It is apparent that the location of the Golgi seen in Fig. 4b cannot be easily distinguished from that of the endoplasmic reticulum in these cells.

Western blot analysis was performed on both $\mathrm{CHO}$ membrane extracts and whole cell extracts to test for the presence of a MC2R-GFP fusion protein in transfected cells. In the absence of an adequate antibody to the MC2R, anti-GFP was used to probe for the presence of a fusion protein. A diffuse band extending between 60 and >105 kDa was seen in MC2R-GFP expressing membranes (Fig. 5a). Since the GFP protein is $\sim 23 \mathrm{kDa}$ in size and the unmodified MC2R is $33 \mathrm{kDa}$, it is highly likely that the composite receptor-GFP molecule has been glycosylated, most probably at the two N-linked glycosylation sites at the $\mathrm{N}$-terminus of the receptor. The smeared appearance of this band is characteristic of a glycoprotein. In addition, the presence of other complexed proteins or even receptor dimers resistant to separation on SDS-PAGE cannot be excluded. It is noticeable that the CCKR-GFP migrates with similar mobility. Both receptor-GFP preparations show a degree of degradation which releases smaller GFP-containing fragments which migrate more slowly than free GFP alone. In view of the 


\section{SK-N-MC}

HEK293

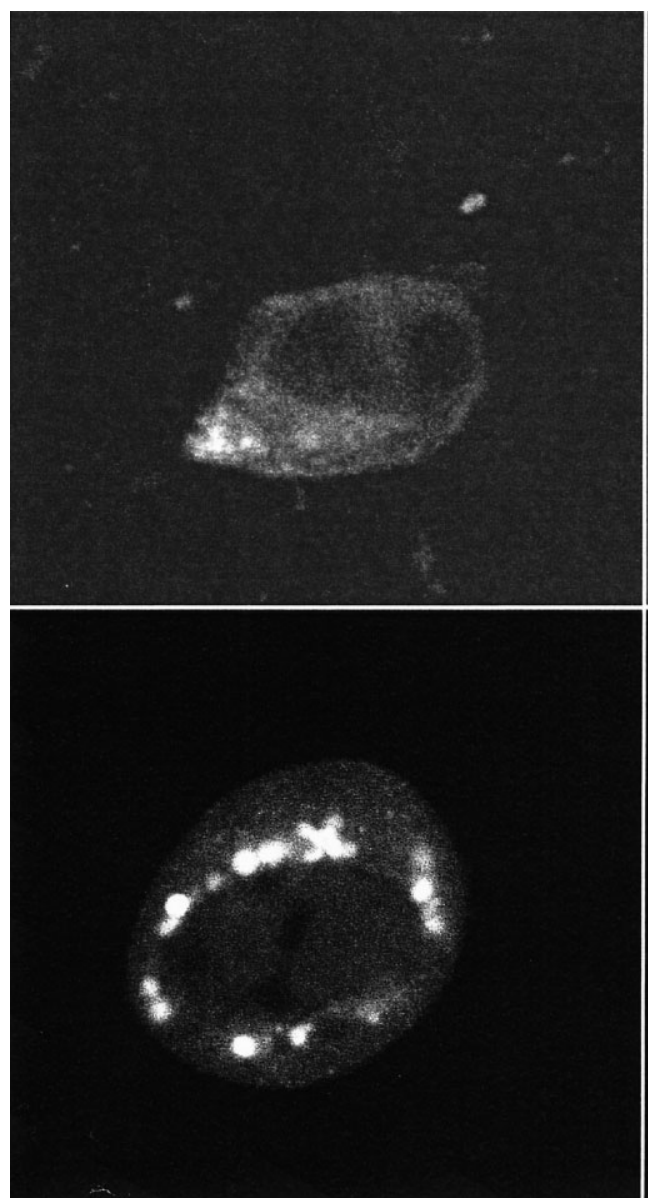

\section{CHO-K1}

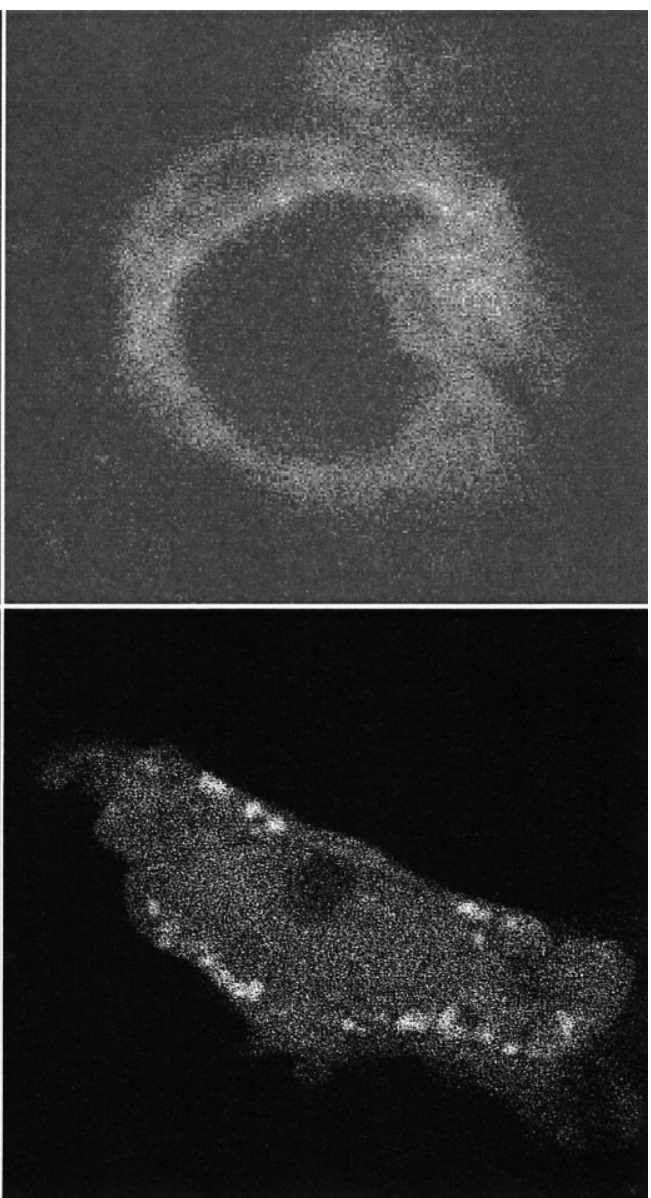

Y6

Figure 3 Fluorescent confocal microscope images of three non-adrenal cell lines (SK-N-MC, HEK293 and CHO-K1 as indicated) and Y6 cells transiently transfected with the MC2R-GFP expression vector. Predominantly perinuclear fluorescence is seen with the three non-adrenal cell types, whereas significant cell surface fluorescence is apparent with the Y 6 cells.

possible existence of non-membrane-associated MC2RGFP in these cells, whole cell extracts were also separated and blotted with the GFP antibody (Fig. 5b). Again, a prominent smeared band of $\sim 60$ to $70 \mathrm{kDa}$ was seen, suggesting the occurrence of effective glycosylation of the MC2R.

In previous studies using GFP-fusion proteins it had been demonstrated that the over-expression of a construct can change the pattern of receptor localization in transfected cells (Tarasova et al. 1997). It was therefore necessary to determine whether or not the observed pattern of fluorescence in the $\mathrm{CHO}$ cell was a physiological phenomenon or a non-specific blockage in the secretory pathway caused by the over expression of the fusion protein. We therefore experimented with the quantity of plasmid DNA and the transfection method used but observed no change in the distribution of fluorescence in $\mathrm{CHO}$ cells (data not shown). In a further experiment, it was found that co-expression of large amounts of wildtype MC2R with CCKR-GFP had no blocking effect on the cell surface trafficking of the CCKR-GFP, a result which would suggest that the observed distribution of GFP in MC2R-GFP transfected cells is the result of a true physiological inability to traffic the receptor.

\section{Discussion}

Since its initial cloning in 1992, characterization of the $\mathrm{MC} 2 \mathrm{R}$ has been severely constrained by the lack of a 
(a)

(b)
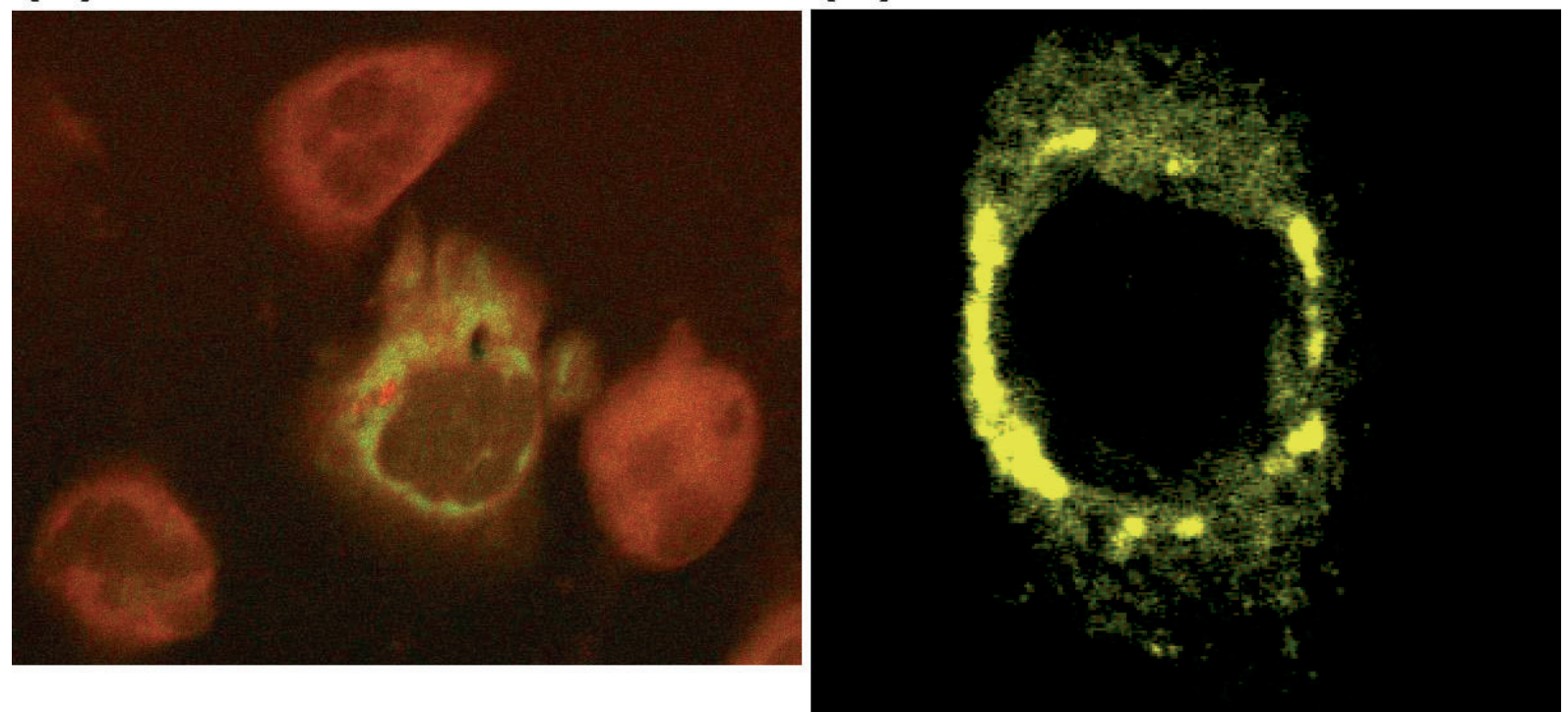

Figure 4 Compartmental localization of MC2R-GFP in CHO cells. (a) Cells were transiently transfected with MC2R-GFP and then immunostained with the TRITC-conjugated anti-KDEL antibody (red). All cells display KDEL immunostaining whereas only a proportion of cells express the transfected plasmid. The cell in the centre of the field shows co-localization of both immunostaining and GFP (yellow). (b) Transient transfection of cells with the Golgi-CFP vector reveals the similar perinuclear localization pattern of the Golgi.

readily transfectable heterologous expression system. Such a system would be of value both for characterization of naturally occurring mutations and for conducting structure-function studies in this receptor that exhibits a very high degree of ligand selectivity. A number of cell lines that express endogenous melanocortin receptors e.g. COS-7 cells and Cloudman S91 cells, can be used to express transfected MC2R, although the presence of the endogenous receptor greatly complicates any functional analysis (Weber et al. 1993, Naville et al. 1996). We previously obtained successful expression data with a single clonal HeLa cell line stably expressing the murine MC2R (Kapas et al. 1996), although our subsequent experience with these cells used for this purpose has been poor, perhaps indicative of a unique feature that had been co-selected in this original cell line. However, use of the Y6 cell (or the closely related OS3 cell) as a host for MC2R expression provides a solution to this problem, although these cells transfect inefficiently, requiring the generation of stably transfected clonal cell lines for receptor characterization (Yang et al. 1997, Elias et al. 1999). This has taken the form of ligand binding studies with $\left[{ }^{125} \mathrm{I}-\right.$ $\left.\mathrm{Phe}^{2}, \mathrm{Nle}^{4}\right]$ ACTH1-24 and ${ }^{125} \mathrm{I}-\mathrm{ACTH} 1-39$ in each study revealing a high affinity site with an $\mathrm{IC}_{50}$ for ACTH of $0.2 \times 10^{-10} \mathrm{M}$. ACTH stimulates cAMP release with an $\mathrm{EC}_{50}$ of $6.8 \times 10^{-9} \mathrm{M}$ (Elias et al. 1999).
The aim of this study was to investigate the mechanism for the failure of expression of MC2R in the non-adrenal cell lines. Theoretically, a defect might lie at several levels. Naville et al. (1996) have reported that transfected MC2R expression vectors are transcribed in that MC2R mRNA is detectable in transfected cells that lack evidence of receptor function. Visualization of the receptor using GFP enables one to confirm its translation and localization of any retardation in the expected progress to the cell surface. However, addition of a large GFP molecule to the receptor protein might adversely influence the normal process of receptor trafficking or folding, and so our initial aim was to demonstrate that in the Y6 cell the presence of GFP does not interfere with receptor function.

Use of a cAMP responsive promoter-reporter such as the $\alpha$ GSU-846 promoter-luciferase construct described here in Y6 cells permits characterization of transiently transfected receptors, and our results with this system confirm the ACTH responsiveness of MC2R-transfected Y6 cells and that an MC2R with a C-terminal GFP tag functions as effectively in $\mathrm{Y} 6$ cells as the wild-type receptor. It is therefore reasonable to conclude that this chimeric molecule is processed and folded correctly and that it acts as a valid marker for MC2R trafficking in the cell. Using this construct we show that the receptor appears to be sequestered in a perinuclear compartment of $\mathrm{CHO}$ cells. A generally similar pattern of fluorescence is observed in other non-adrenal cell lines including 
HEK293 and SK-N-MC cells. In contrast, when the GFP-tagged MC2R is expressed in Y6 cells, a distinct pattern of expression with significant cell surface fluorescence is found.

Co-localization of MC2R-GFP fluorescence with KDEL immunofluorescence supports the conclusion that the bulk of the receptor is retained within the endoplasmic reticulum in $\mathrm{CHO}$ cells. An alternative or additional possibility is that the receptor is retained in the Golgi apparatus, and the pattern of fluorescence generated by the EYFP-Golgi marker seen in Fig. 4b suggests a comparable localization pattern in the $\mathrm{CHO}$ cell. Thus the possibility must remain that the block in trafficking lies in the Golgi, and the receptor accumulates upstream of this in the endoplasmic reticulum. Immunoblot studies using the GFP antibody suggest that the hybrid protein is both membrane associated and glycosylated in $\mathrm{CHO}$ cells - a finding that is consistent with residence in or beyond the endoplasmic reticulum. Export from this site has been identified as the limiting step in the processing of several GPCRs including the $\delta$ opioid receptor (Petaja-Repo et al. 2000) and the gonadotrophin receptor (Rozell et al. 1998). Our data imply that this is a specific process rather than a non-specific 'clogging' of trafficking channels in that co-expression of the MC2R and the CCKR-GFP does not impair cell surface expression of the latter.

There are many examples of inefficient trafficking of transfected GPCRs. Studies with Drosophila rhodopsins have identified a specific role for a cyclophilin-like gene, ninaA (Colley et al. 1991). In common with other cyclophilins, nina $A$ has peptidyl-prolyl cis-trans isomerase activity enabling it to assist in rhodopsin folding in the endoplasmic reticulum. nina $A$ mutants result in retention of rhodopsin in the endoplasmic reticulum (Baker et al. 1994). Mammalian homologues of ninaA have been identified and these are expressed in a tissue-specific manner in retinal cells (Ferreira et al. 1995). Caenorhabditis elegans olfactory receptors are localized to the cilia of olfactory neurones under the direction of the odr-4 and odr- 8 genes. These genes encode proteins that may also act as chaperones, assisting folding of the receptor, although alternative functions such as sorting or targeting of receptors has been considered (Dwyer et al. 1998, Gimelbrandt et al. 1999). $o d r-4$ and odr-8 have no homology to nina $A$ or other cyclophilins. Interactions between the $\mathrm{N}$ - and C-terminal domains of the olfactory receptors appear to be involved in this process (Gimelbrandt et al. 1999). Recently, Bermak et al. (2001) have identified a novel protein, DRiP78, that binds the C-terminus of the D1 dopamine receptor in the endoplasmic reticulum and acts to regulate export of this and potentially other GPCRs from this site. Ho and MacKenzie (1999) have shown a crucial endoplasmic reticulum export role for the C-terminal region of the melanocortin 4 receptor, which has significant structural and functional similarity to the MC2R. (a)

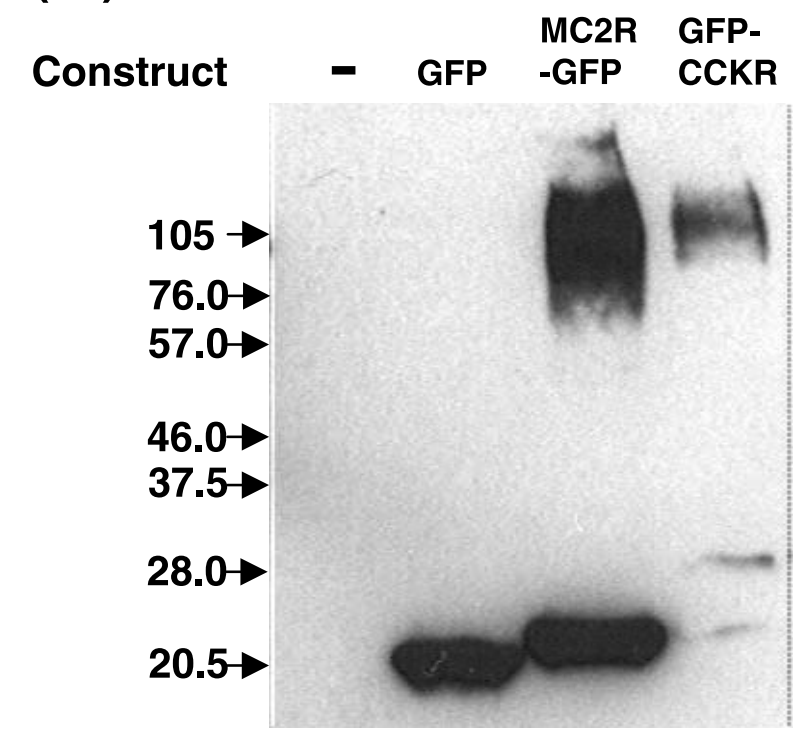

(b)

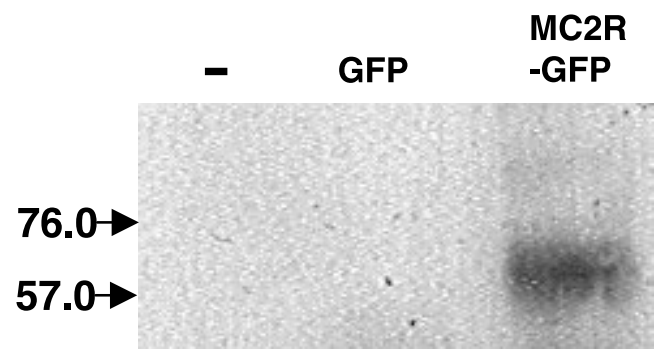

Figure 5 Immunoblot of MC2R-GFP using the GFP antibody on (a) $\mathrm{CHO}$ membrane extracts prepared as by Pryde (1998) using Triton X-114 and separated on SDS-PAGE, showing cells either untransfected $(-)$, or transfected with pEGFP (GFP), the MC2R-GFP or the CCKR-GFP. (b) CHO cells extracted according to Tolwinski et al. (1999) and separated on SDS-PAGE showing untransfected cells $(-)$, pEGFP transfected cells (GFP) or MC2R-GFP transfected cells.

The example of the receptor activity modifying proteins (RAMPs) is particularly interesting. Early attempts at expression cloning of the calcitonin gene-related peptide (CGRP) receptor were impeded because of difficulties in obtaining functional expression. The calcitonin receptorlike receptor (CRLR) was originally cloned on the basis of its homology to the calcitonin receptor (CT-R) (Chang et al. 1993, Njuki et al. 1993, Muff et al. 1995). Expression studies, with one exception (Aiyar et al. 1996), failed to identify a CGRP binding or signalling role and it was therefore designated an orphan receptor. Subsequently, McLatchie et al. (1998) demonstrated that coexpression of the CRLR with one of three RAMPs conferred function as 
either a CGRP receptor or an adrenomedullin receptor, depending on the RAMP expressed. The RAMP proteins appear to be multifunctional and are essential for the transport of the receptor from the endoplasmic reticulum to the cell surface, for specific glycosylation, for ligand binding specificity (McLatchie et al. 1998, Fraser et al. 1999) and possibly for receptor desensitization (Drake et al. 1999, 2000). Furthermore, it appears that the RAMPs have a similar role in modulating the function of the CT-R as a receptor for calcitonin, amylin or CGRP (Muff et al. 1999).

We hypothesize that an accessory factor is also required for expression of the MC2R. Such a factor appears to be specific to cells of adrenal origin, or possibly to cells that express a member of the melanocortin receptor family, a feature shared by bovine nina $A$ homologues. The role of such a factor would probably be that of a chaperone in ensuring correct folding of this receptor.

A question raised by these reports is why certain receptors may have this requirement for accessory factors while others apparently do not. It is conceivable, however, that the efficiency of the majority of transfection/ expression systems obscures the relative inefficiencies of GPCR expression in the natural state. mRNA levels for many endogenous GPCRs are very low and considerably less than those obtained in cells transfected with most receptor expression vectors, perhaps suggesting that each of these receptors requires some form of post-translational accessory factor. Alternatively, it may be that many GPCRs make use of common, ubiquitous accessory factors, the role of which is thus difficult to detect. Identification of the putative MC2R accessory factor will be of great interest both from the cell biological point of view and as a candidate factor for human inherited ACTH insensitivity. $\mathrm{Up}$ to a half of such cases result from mutations of the $\mathrm{MC} 2 \mathrm{R}$, whereas the remainder are linked to alternative genetic loci (Clark \& Weber 1998). Defects in this accessory factor would provide a highly credible candidate gene for this syndrome.

In summary, we have shown that a C-terminal GFPtagged $\mathrm{MC} 2 \mathrm{R}$ migrates to the cell surface and retains function in an adrenal-derived cell, but is retarded in the endoplasmic reticulum/Golgi in other cell types. This retardation is specific to this receptor and the CCKR can move through the endoplasmic reticulum in the presence of MC2R without difficulty. These findings are highly suggestive of a tissue-/cell-specific accessory factor that the $\mathrm{MC} 2 \mathrm{R}$ requires for full expression in the adrenocortical cell. Attempts to characterize and clone this factor are currently underway.

\section{Acknowledgements}

We are very grateful to Prof. Bernard Schimmer (University of Toronto) for providing the Y6 cell line, to Dr S A Wank (NIDDK, NIH) for the use of the CCKR-GFP expression vector, to Prof. J Burrin and Dr R Fowkes (Queen Mary, University of London) for the $\alpha$-GSUluciferase reporter, and to Prof. J Priestley (Queen Mary, University of London) and Dr F A Meunier (Imperial Cancer Research Fund, Lincoln's Inn, London) for advice on confocal microscopy techniques. This work was supported by a project grant from the Joint Research Board of St Bartholomew's Hospital.

\section{References}

Aiyar N, Rand K, Elshourbagy NA, Zeng Z, Adamou JE, Bergsma DJ \& Li Y 1996 A cDNA encoding the calcitonin gene-related peptide type 1 receptor. Journal of Biological Chemistry 271 11325-11329.

Baig AH, Swords FM, Noon L, King PJ, Hunyady L \& Clark AJL 2001 Desensitization of the Y1 cell adrenocorticotropin receptor: evidence for a restricted heterologous mechanism implying a role for receptor-effector complexes. Journal of Biological Chemistry 276 $44792-44797$.

Baker EK, Colley NJ \& Zuker CS 1994 The cyclophilin homolog NinaA functions as a chaperone, forming a stable complex in vivo with its protein target rhodopsin. EMBO Journal 13 4886-4895.

Barak LS, Ferguson SS, Zhang J, Martenson C, Meyer T \& Caron MG 1997 Internal trafficking and surface mobility of a functionally intact beta2-adrenergic receptor-green fluorescent protein conjugate. Molecular Pharmacology 51 177-184.

Bermak JC, Li M, Bullock C \& Zhou Q-Y 2001 Regulation of transport of the dopamine D1 receptor by a new membraneassociated ER protein. Nature Cell Biology 3 492-497.

Burrin JM, Aylwin SJ, Holdstock JG \& Sahye U 1998 Mechanism of action of pituitary adenylate cyclase-activating polypeptide on human glycoprotein hormone alpha-subunit transcription in alphaT3-1 gonadotropes. Endocrinology 139 1731-1737.

Chang CP, Pearse RV II, O’Connell S \& Rosenfeld MG 1993 Identification of a seven transmembrane helix receptor for corticotropin-releasing factor and sauvagine in mammalian brain. Neuron 11 1187-1195.

Clark AJL \& Weber A 1998 Adrenocorticotropin insensitivity syndromes. Endocrine Reviews 19 828-843.

Colley NJ, Baker EK, Stamnes MA \& Zuker CS 1991 The cyclophilin homolog ninaA is required in the secretory pathway. Cell 67 255-263.

Drake WM, Ajayi A, Lowe S, Mirtella A, Bartlett TJ \& Clark AJL 1999 Desensitization of CGRP and adrenomedullin receptors in SK-N-MC cells: implications for the RAMP hypothesis. Endocrinology 140 533-537.

Drake WM, Lowe SR, Mirtella A, Bartlett TJ \& Clark AJL 2000 Desensitisation of calcitonin gene-related peptide responsiveness but not adrenomedullin responsiveness in rat vascular smooth muscle cells. Journal of Endocrinology 165 133-138.

Dwyer ND, Troemel ER, Sengupta P \& Bargmann CI 1998 Odorant receptor localization to olfactory cilia is mediated by ODR-4, a novel membrane-associated protein. Cell 93 455-466.

Elias LLK, Weber A, Pullinger GD, Mirtella A \& Clark AJL 1999 Functional characterization of naturally occurring mutations of the human adrenocorticotropin receptor: poor correlation of phenotype and genotype. Journal of Clinical Endocrinology and Metabolism $\mathbf{8 4}$ 2766-2770.

Ferreira PA, Hom JT \& Pak WL 1995 Retina-specifically expressed novel subtypes of bovine cyclophilin. Journal of Biological Chemistry $27023179-23188$

Fraser NJ, Wise A, Brown J, McLatchie LM, Main MJ \& Foord SM 1999 The amino terminus of receptor activity modifying proteins is a critical determinant of glycosylation state and ligand binding of 
calcitonin receptor-like receptor. Molecular Pharmacology $\mathbf{5 5}$ 1054-1059.

Frigeri C, Tsao J, Czerwinski W \& Schimmer BP 2000 Impaired steroidogenic factor 1 (NR5A1) activity in mutant Y1 mouse adrenocortical tumor cells. Molecular Endocrinology 14 535-544.

Gimelbrandt AA, Stoss TD, Landers TM \& McClintock TS 1999 Truncation releases olfactory receptors from the endoplasmic reticulum of heterologous cells. Journal of Neurochemistry $\mathbf{7 2}$ 2301-2311.

Ho G \& MacKenzie RG 1999 Functional characterization of mutations in the melanocortin-4 receptor associated with human obesity. Journal of Biological Chemistry 274 35816-35822.

Kallal L \& Benovic JL 2000 Using green fluorescent proteins to study G protein-coupled receptor localization and trafficking. Trends in Pharmacological Sciences 21 175-180.

Kapas S, Cammas FM, Hinson JP \& Clark AJL 1996 Agonist and receptor binding properties of adrenocorticotropin peptides using the cloned mouse ACTH receptor expressed in a stably transfected HeLa cell line. Endocrinology 137 3291-3294.

McLatchie LM, Fraser NJ, Main MJ, Wise A, Brown J, Thompson N, Solari R, Lee MG \& Foord SM 1998 RAMPs regulate the transport and ligand specificity of the calcitonin receptor-like receptor. Nature 393 333-339.

Mountjoy KG, Robbins LS, Mortrud MT \& Cone RD 1992 The cloning of a family of genes that encode the melanocortin receptors. Science 257 1248-1251.

Muff R, Hunziker W, Fischer JA \& Born W 1995 A human orphan calcitonin receptor-like structure. Biochemical and Biophysical Research Communications 206 341-347.

Muff R, Buhlmann N, Fischer JA \& Born W 1999 An amylin receptor is revealed following co-transfection of a calcitonin receptor with receptor activity modifying proteins- 1 or -3 . Endocrinology $1402924-2927$.

Naville D, Barjhoux L, Jaillard C, Faury D, Despert F, Esteva B, Durand P, Saez JM \& Begeot M 1996 Demonstration by transfection studies that mutations in the adrenocorticotropin receptor gene are one cause of the hereditary syndrome of glucocorticoid deficiency. Journal of Clinical Endocrinology and Metabolism 81 1442-1448.

Njuki F, Nicholl CG, Howard A, Mak JC, Barnes PJ, Girgis SI \& Legon S 1993 A new calcitonin-receptor-like sequence in rat pulmonary blood vessels. Clinical Science 85 385-388.

Penhoat A, Naville D, El Mourabit H, Buronfosse A, Durand P \& Begeot M 2000 Functional expression of the human ACTH receptor gene. Endocrine Research 26 549-557.

Petaja-Repo UE, Hogue M, Laperriere A, Walker P \& Bouvier M 2000 Export from the endoplasmic reticulum represents the limiting step in the maturation and cell surface expression of the human delta opioid receptor. Journal of Biological Chemistry 275 13727-13736.
Pryde JG 1998 Partitioning of proteins in Triton X-114. In Methods in Molecular Biology 88 pp 23-33. Ed. RA Clegg. Totowa, NJ, USA: Humana Press.

Rozell TG, Davis DP, Chai Y \& Segaloff DL 1998 Association of gonadotropin receptor precursors with the protein folding chaperone calnexin Endocrinology 139 1588-1593.

Schimmer BP, Kwan WK, Tsao J \& Qiu R 1995a Adrenocorticotropinresistant mutants of the Y1 adrenal cell line fail to express the adrenocorticotropin receptor. Journal of Cell Physiology 163 164-171.

Schimmer BP, Kwan WK, Tsao J \& Qiu R 1995b ACTH-receptor deficient mutants of the $\mathrm{Y} 1$ mouse adrenocortical tumor cell line. Endocrine Research 21 139-156.

Schulein R, Lorenz D, Oksche A, Wiesner B, Hermosilla R, Ebert J \& Rosenthal W 1998 Polarized cell surface expression of the green fluorescent protein-tagged vasopressin V2 receptor in Madin Darby canine kidney cells. FEBS Letters 441 170-176.

Tang BL, Wong SH, Qi XL, Low SH \& Hong W 1993 Molecular cloning, characterization, subcellular localization and dynamics of p23, the mammalian KDEL receptor. Journal of Cell Biology 120 325-328.

Tarasova NI, Stauber RH, Choi JK, Hudson EA, Czerwinski G, Miller JL, Pavlakis GN, Michejda CJ \& Wank SA 1997 Visualization of $\mathrm{G}$ protein-coupled receptor trafficking with the aid of the green fluorescent protein. Endocytosis and recycling of cholecystokinin receptor type A. Journal of Biological Chemistry 272 14817-14824.

Tavare JM, Fletcher LM \& Welsh GI 2001 Using green fluorescent protein to study intracellular signalling. Journal of Endocrinology 170 297-306.

Tolwinski NS, Shapiro PS, Goueli S \& Ahn NG 1999 Nuclear localization of mitogen-activated protein kinase kinase 1 (MKK1) is promoted by serum stimulation and G2-M progression. Requirement for phosphorylation at the activation lip and signaling downstream of MKK. Journal of Biological Chemistry 274 6168-6174.

Weber A, Kapas S, Hinson J, Grant DB, Grossman A \& Clark AJL 1993 Functional characterization of the cloned human ACTH receptor: impaired responsiveness of a mutant receptor in familial glucocorticoid deficiency. Biochemical and Biophysical Research Communications 197 172-178.

Yang YK, Ollmann MM, Wilson BD, Dickinson C, Yamada T, Barsh GS \& Gantz I 1997 Effects of recombinant agouti-signaling protein on melanocortin action. Molecular Endocrinology 11 274-280.

Received 4 March 2002

Accepted 22 March 2002 